\title{
Fat Loss in Stored, Refrigerated/Thawed Expressed Breast Milk
}

\author{
KE ELIZABETH \\ Professor of Pediatrics and Superintendent, SAT Hospital, Govt. Medical College, Thiruvananthapuram 695011 \\ elizake@hotmail.com
}

B reastmilk is a life savior to millions of young children across the globe. Breast milk ensures physical, mental and emotional wellbeing. As per the 'Infant and Young Child Feeding' (IYCF) concepts, nutrition of the first 1000 days of life, which includes 9 months of intra uterine period (270 days) to 24 months post natal period ( 730 days), lays the foundation for the nutritional and health status of an individual. Nature has assigned the responsibility of this period solely on the mother. Among the IYCF practices, an important bottle neck is suboptimal breastfeeding due to various reasons including employment or other commitments of mothers. Expressed breast milk (EBM) being the mainstay in the feeding of preterm and low birth weight (LBW) babies and with several mothers going out for work, there is need for storing and thawing. As milk expands on freezing, the container should not be full during storage. EBM should be stored in clean, capped glass or hard polypropylene plastic containers or special freezing bags.EBM should not be stored in the door and in disposable bottles or feeding bottles or plastic bags designed for general household use. Before expressing or handling breast milk, hands should be washed with soap and water. Fresh breast milk is always superior as storage and thawing decreases fat, certain vitamins, cellular and host defense properties of the milk. Fat can adhere to plastic container and tubing, and cellular components to glass. At room temperature, fat is broken down to triglycerides which change the $\mathrm{pH}$ of milk and turn it sour. This is different from the problem of bacterial contamination. Actually, the bacteria will be less in number at six hours than they were at the time of pumping because of the protective qualities in the milk, which suppress bacterial growth. On the other hand, the enzymes decline later and bacterial counts also start to rise [1].

For thawing frozen EBM, it should be placed in the refrigerator the night before, followed by gentle rewarming of the container in a bowl of warm water or holding under warm running water [2]. Heating and microwaving are not recommended due to uneven heating and loss of anti-infective properties. Milk separates during storage and fat rises to the top. Do not refreeze milk once it has been thawed or partially thawed. It is prudent to note that thawed breast milk should be used within 24 hours and any remaining milk should be discarded. Some mothers have a high level of lipase enzyme and their milk may smell and taste soapy when thawed. This milk is not harmful, but the baby may sometimes refuse it.

Freezing, storing and thawing are known to result in loss of fat, and thereby energy [3]. Even in fresh breast milk, the lactose and protein content remain relatively constant, but a variation is noted in the fat content. Thawing of frozen breast milk results in separation of fat and a decrease in bile salt dependent lipase, vitamins $C$, $\mathrm{B}_{6}$ and folacin, that are heat labile [4]. Pasteurization $\left(62.5^{\circ} \mathrm{C} \times 30 \mathrm{~min}\right)$ does not alter fatty acid composition of milk, but sterilization $\left(120^{\circ} \mathrm{C} \times 30 \mathrm{~min}\right)$ results in up to $13 \%$ fat loss and also linoleic and arachidonic acid [5]. At the melting point of fat, solid fat turns to oil fat and oil can adhere to the sides of the container. In the comparative study of milk using fresh milk (FM) and thawed frozen milk by way of overnight refrigeration (RM) and warming in warm water (WM), the authors have reported that RM retains better fat content than WM [6].

The information that thawing frozen breast milk by leaving it in refrigerator compartment the night before is a better method to preserve quality and energy than warming by placing in warm water needs to be passed on 'from the bench to bedside', as refrigerators are now an integral part of all households.

Funding: None; Competing interests: None stated.

\section{REFERENCES}

1. Hamosh M, Ellis LA, Pollock DR, Henderson TR, Hamosh P. Breast feeding and other working mothers: effect of time and temperature of short term storage on proteolysis, lipolysis and bacterial growth in milk. Pediatrics. 1996;97:492-8.

2. American Academy of Pediatrics. Breast feeding. In: Kleinmann RE, editor. Pediatric nutrition handbook. $6^{\text {th }}$ ed. Elk Grove Village: American Academy of Pediatrics. 2009. P.51-60. 
3. Berkow SE, Freed LM, Hamosh M, Bitman J, Wood DL, Happ B et al. Lipases and lipids in human milk; effect of freeze- thawing and storage. Pediatr Res. 1984;18: 1257-62.

4. Lavine M, Clark RM. Changing pattern of FFA in breastmilk during storage. J Pediatr Gastroenterol Nutr. 1987;6:769-74.
5. Fidler N, Sauerwald TU, Koletzko B, Demmelmair H. Effects of human milk pasteurization and sterilization on available fat content and fatty acid composition. J Pediatr Gastroenterol Nutr. 1998;132:876-8

6. Thatrimontrichai A, Janjindamai W, Puwanant M. Fat loss in thawed breast milk: Comparison between refrigerator and warm water. Indian Pediatr. 2012;49:877-80 .

\title{
Disease Course in Childhood Steroid Sensitive Nephrotic Syndrome: Is it Changeable?
}

\author{
SHUICHI Ito \\ Division Chief, Division of Nephrology and Rheumatology, National Center for Child Health and Development, \\ 2-10-1 Okura, Setagaya-ku, Tokyo, 157-8535. \\ ito-shu@ncchd.go.jp
}

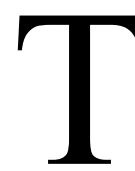

he long-term prognosis of steroid-sensitive nephrotic syndrome is relatively good. However, most patients experience relapses in their clinical course. In this issue, Sinha, et al.

[1] report the prognosis of steroid-sensitive nephrotic syndrome in 1071 children, which is the largest retrospective cohort study to date. They describe that approximately one-half of the patients developed frequent-relapsing nephrotic syndrome (FRNS) or steroid-dependent nephrotic syndrome (SDNS), consistent with the rates in previous reports. FRNS and SDNS are critical issues for children, because of the severe adverse effects of steroid treatment, and psychological issues owing to long hospitalization, frequent hospital visits, and side effects of treatment. Therefore, clarification of risk factors for a frequentrelapsing course and its prevention are quite important. The authors concluded that early onset ( $<4$ years), lack of adequate initial therapy ( $<8$ weeks), and short duration of initial remission lasting $<6$ months are risk factors for a frequent-relapsing course. Among these, the only one in which we can intervene is initial therapy. Although many pediatric nephrologists think that initial steroid treatment at disease onset may influence the future clinical course, the optimal length of initial steroid therapy remains controversial. A standard basic initial steroid therapy at primary onset was proposed by the International Study of Kidney Disease in Children (ISKDC) more than 30 years ago. The proposal was daily steroid therapy $\left(60 \mathrm{mg} / \mathrm{m}^{2} / \mathrm{d}\right)$ for 4 weeks followed by alternate-day treatment $(40 \mathrm{mg} /$ $\mathrm{m}^{2} / 2 \mathrm{~d}$ ) for 4 weeks (original was 3 days of medication followed by 4 days off in a week, but later modified). Thereafter, this protocol was revised in a variety of ways to obtain a better prognosis.
The KDIGO (Kidney Disease: Improving Global Outcomes) guideline recommends that oral prednisone should be administered as a single daily dose starting at $60 \mathrm{mg} / \mathrm{m}^{2} / \mathrm{d}$ or $2 \mathrm{mg} / \mathrm{kg} / \mathrm{d}$ (maximum: $60 \mathrm{mg} / \mathrm{d}$ ) for $4-6$ weeks followed by alternate-day medication at $40 \mathrm{mg} / \mathrm{m}^{2} /$ $2 \mathrm{~d}$ or $1.5 \mathrm{mg} / \mathrm{kg} / 2 \mathrm{~d}$ (maximum: $40 \mathrm{mg} / 2 \mathrm{~d}$ ) with tapering of the dose for 2-5 months [2]. The published US guideline recommends that the initial steroid therapy should be $2 \mathrm{mg} / \mathrm{kg} / \mathrm{d}$ for 6 weeks followed by $1.5 \mathrm{mg} / \mathrm{kg} /$ $2 \mathrm{~d}$ on alternate days for 6 weeks (maximum: $40 \mathrm{mg} / 2 \mathrm{~d}$ ) [3]. In the Cochrane systematic review, it is stated that duration of therapy up to 7 months appeared to be more effective than therapy for 2 months in achieving sustained remission. In Japan, a randomized controlled trial (RCT) comparing daily therapy for 4 weeks followed by alternate-day therapy for 6 months with the standard 2month therapy is ongoing. The results of this RCT will provide some suggestions regarding the optimal mode of initial steroid treatment. The duration of daily steroid therapy, i.e. 4 or 6 weeks, should also be evaluated by an RCT.

Although half of the 1071 patients progressed to FRNS, 185 children had FRNS or SDNS and 42 had late steroid resistance in long-term observation. However, $72 \%$ of FRNS patients were in remission or had infrequent relapses with immunosuppressive agents or low-dose steroid. Therefore, the long-term prognosis is relatively good. However, a certain proportion of patients showed disease transition to the adult form or a difficult clinical course resistant to existing therapy. Such patients were likely to be complicated with severe infection, resulting in high mortality in their study. Recently, rituximab, an anti-CD20 antibody, has become an 